Background/introduction Homeless adults and street based sex workers are a highly vulnerable group of people with specific sexual and general health needs. A specialist outreach clinic was set up in 2010 to support these patients.

Aim(s)/objectives To evaluate the uptake of services used including contraception, immunisation, blood-borne virus testing, cytology, STI screening and evaluation of drug use.

Methods Data was retrospectively collected from May 2012 until March 2015.

Results 82 patients seen in total (female, 53; male, 29), with an average age of 28.6 (range 17-50.) 57\% of patients were symptomatic. $57 \%$ patients $(\mathrm{n}=47)$ were Hepatitis B immune, $26 \%$ $(\mathrm{n}=21)$ received either boosters or full vaccination for HBV. $34 \%$ patients $(\mathrm{n}=28)$ had STIs. Hepatitis C $(36 \%)$ and Chlamydia (32\%) were the most common infections. 57\% patients ( $\mathrm{n}=47$ ) were using drugs, the majority using heroin (57\%). 3 females were pregnant at baseline review; of the remaining women, $78 \%(\mathrm{n}=39)$ were on contraception, LARCs being the most widely used. $34 \%$ of women $(n=18)$ were working as commercial sex workers. 35 of the women had given birth to a total of 97 children, with $70 \%$ of them $(n=68)$ either fostered or adopted. $33 \%$ smears taken $(n=10)$ were abnormal with 3 colposcopy referrals.

Discussion/conclusion This specialist outreach clinic facilitates sexual and reproductive healthcare for vulnerable patients who are otherwise hard to reach and often have poor experiences of healthcare. The high rate of sex work in this population emphasises the need for continued screening and treatment. LARC uptake rates are reassuring, but could be further improved.

\section{P007 TRIALS AND TRIBULATIONS-CREATING A SEXUAL HEALTH LEAFLET FOR PRISONERS}

${ }^{1}$ Jennifer Murira*, ${ }^{2}$ Paul Moore. 'Leeds Teaching Hospitals Trust, Leeds, UK; ${ }^{2}$ Public Health England (Yorkshire and Humber branch), Leeds, UK

10.1136/sextrans-2016-052718.62

Background/introduction Her Majesty's Inspectorate of Prisons recommends that prisoners are provided with sexual health information and condoms. Consensual sex rates for prisoners are reported between 1.6-10\%, and they are considered high risk for STI's.

Aim(s)/objectives A request by a prison healthcare team for a sexual health leaflet prompted the creation of a pamphlet specific for prisoners.

Methods A working group was created between Genitourinary medicine, Public health England and prison healthcare. A literature review was conducted on sexual/prison healthcare leaflets. 30 prisoners were consulted informing content, length and language. A draft was given to a second focus group who completed a questionnaire to evaluate the impact of the leaflet. Approval of the content and look were required from the prison governor. Decisions were needed regarding dissemination and costs.

Results Literature review revealed no previous leaflet for prisoners on sexual health. Prisoners highlighted eye-catching language, pictures,'reference' style and a quiz being important points that would increase use of a leaflet. A second focus group questionnaire indicated the draft leaflet increased their knowledge about sexual health (90\%) and would make them much more likely to wear a condom (52\%). Difficulties arose around language used within the leaflet particularly the title acceptability to prison staff and who would fund printing costs. This impacted on distribution and reach of the leaflet.

Discussion/conclusion A simple request lead to a complex lengthy solution, many parties required consultation with differing views. Finally, we hope to have created a leaflet that is applicable for all prisons across England.

\section{P008 AN AUDIT OF TIME TO TREATMENT FOR BACTERIAL STIS, AND TIME TO PROVISION OF HIV DIAGNOSIS, IN A LARGE URBAN SEXUAL HEALTH CLINIC}

${ }^{1}$ Anthony Snow*, ${ }^{1}$ Ria Fortune, ${ }^{1,2}$ Marcus Chen, ${ }^{1,2}$ Christopher Fairley, ${ }^{1,3}$ David Lee. ${ }^{1}$ Melbourne Sexual Health Centre, Melbourne, Victoria, Australia; ${ }^{2}$ Monash University, Melbourne, Victoria, Australia; ${ }^{3}$ Melbourne Univeristy, Melbourne, Victoria, Australia

\subsection{6/sextrans-2016-052718.63}

Background The time from testing to treatment of STIs, and the provision of a new HIV diagnosis", is a marker of quality of care. The follow-up of positive results is undertaken by nurses according to predetermined protocols. In April 2015 gonococcal NAAT superseded the relatively insensitive gonococcal culture test.

Aims The aims were to determine the time to treatment for HIV $^{*}$, syphilis, gonorrhoea and chlamydia; and if the introduction of gonorrhoea NAAT affected the time to treatment.

Methods This observational study compared the median time (days) to treatment for HIV* and STIs in two time periods (P1: April-June 2014 and P2: April-June 2015). For gonorrhoea, the median time from testing to result complete and median followup time to treatment were also compared. The Mann-Whitney U Test for two independent samples was used to compare medians. Results The median time to treatment for all STIs, including HIV $^{*}$, was 8 days or less in P1 and P2 (all p $\geq 0.08$ ). The time to result complete for gonorrhoea was significantly less in P2 (n $=189$, median $=3)$ compared to $\mathrm{P} 1(\mathrm{n}=50$, median $=5)$ $(\mathrm{p}=0.000)$. However, the median follow-up time to treatment was not significantly different between P1 (median $=3$ ) and P2 $($ median $=4)(\mathrm{p}=0.4)$.

Discussion/Conclusion The median time to treatment for HIV*, syphilis, gonorrhoea and chlamydia was not significantly different between P1 and P2. Despite gonorrhoea NAAT results being available significantly earlier, the overall time to treatment was not different. This likely relates to the nearly fourfold increase in the detection of gonorrhoea and the additional burden of work for follow-up nurses.

\section{P009 A REVIEW OF SEXUAL HEALTH CARE ACCESS AND OUTCOMES AMONG WOMEN WHO HAVE SEX WITH WOMEN}

Emma Dorothy Mills. University of Aberdeen, Aberdeen, UK

\subsection{6/sextrans-2016-052718.64}

Background/introduction Women who have sex with women (WSW) are at risk of sexual ill-health, yet health professionals are ill-informed regarding the range of sexual health issues affecting these women. This ignorance may compound 
misconceptions among WSW regarding their risk status and the services available to them. Sexually transmitted infections (STIs), abnormal cervical cytology, and unplanned pregnancy are conditions which are crucially exacerbated by barriers to engagement with health care.

Aim(s)/objectives To review the recent literature regarding access to sexual health care among WSW, and discuss some of the indicators of sexual ill-health adversely impacted by barriers to such engagement.

Methods Relevant databases (MEDLINE, Embase) were searched using $\mathrm{MeSH}$ terms related to sexual health, engagement with health serves, and WSW.

Results This review demonstrates that WSW experience several barriers to care, including the heteronormative expectations of health professionals. Studies suggest the prevalence of STIs among WSW is comparable to heterosexual women, while the use of barrier protection is limited. Screening uptake for cervical cancer among WSW remains poor. In addition, sexual minority, sexually active young women are more likely to experience an unplanned pregnancy than their heterosexual peers.

Discussion/conclusion The findings with regards to STI risk and unplanned pregnancy highlight the need for targeted interventions to address sexual risk taking behaviour among WSW. Further research should be conducted to examine the effectiveness of such interventions. Furthermore, greater understanding of the sexual health of WSW is urgently required among clinicians to improve care and engagement with healthcare among this population of women.

\section{P010 MAXIMISING DEPARTMENTAL INCOME; A QUALITY IMPROVEMENT PROJECT FOR IMPROVING HIV TESTING AND CODING}

Laura Parry. Barts NHS Trust, London, UK

\subsection{6/sextrans-2016-052718.65}

Background BASHH standards recommend that $97 \%$ of $1^{\text {st }}$ attenders to GUM services should be offered an HIV test to facilitate prompt diagnosis. This standard now features as a key performance indicator (KPI) in contracts where financial penalties are imposed for non-compliance.

Aim 97\% of first GUM attendances will be offered an HIV test by August 2015.

Methods Quality improvement (QI) methodology was applied and key drivers were identified: 1) Staff: Timetabled administration sessions and training. 2) Communication: Weekly email reminders to staff regarding coding accuracy. 3) Timing: Timely upload of missed HIV codes by reception. 4) Measurement: Performance recorded/reviewed monthly.

Plan-do-study-act cycles (PDSA) were used PDSA 1: Computerised administration recall system launched resulting in all clinical administration tasks becoming computerised and accessible from any site across the Trust. Standard Operating Procedures (SOP) developed. Team training. PDSA 2: Reception team briefed/delegated task of uploading missed HIV codes. Weekly email reminders sent to staff. PDSA 3: Administration recall SOPs uploaded to intranet. New staff inductions delivered.

Results Prior to introduction of this project only $89 \%$ of new attenders were offered an HIV test (May 2014). We have exceeded our aim with $100 \%$ offered, avoiding a potential penalty of $£ 19,165$ per month, securing $£ 229,980$ income over the past 12 months.

Discussion Using QI methodology, robust systems can be implemented improving patient care and facilitating meeting KPIs.

\section{P011 IMPROVING TIME TO TREATMENT; A QUALITY IMPROVEMENT PROJECT FOR RESULTS HANDLING OF NON-STANDARD GUM TESTS}

Laura Parry. Barts NHS Trust, London, UK

\subsection{6/sextrans-2016-052718.66}

Background Delays to treatment following late non-standard results (NSR) review (e.g. mid-stream urine or radiological tests) by a doctor can cause patient harm. There are on average 10 NSR per week in our department. Prior to this project there was limited governance around clinician review of results with most done in an adhoc way sometimes causing significant delays to treatment $(2+$ weeks). Verbal communication with staff often did not result in NSR being actioned faster. Patients would often make multiple calls to the results team resulting in poor patient experience.

Aim All NSR, once available, will be actioned within 7 days by August 2015.

Methods Quality improvement (QI) methodology applied and key drivers identified: 1) Staff: Training, timetabled administration sessions. 2) Communication: Clear roles/responsibilities identified, email communication. 3) Timing: Timely upload of NSR onto recall list by results team. 4) Measurement: Recall list checked daily, NSR remaining recorded.

Plan-do-study-act cycles (PDSA) were used over six months PDSA 1: Developed a computerised recall system. Standard Operating Procedures (SOP) written. Team training. PDSA 2: Results team briefed/delegated task of recording remaining NSR. PDSA 3: SOPs uploaded to intranet. Email communication with new staff. SHO induction briefing (every four months).

Results We now have on average only one outstanding NSR per week. Verbal communication from the results team has confirmed much improved patient satisfaction.

Discussion Through QI methodology and the development of a simple organised governance system, patient care and satisfaction can be improved. Additional PDSA cycles are planned to further service improvement.

\section{P012 MANAGING MYCOPLASMA GENITALIUM: ARE WE DOING ENOUGH?}

${ }^{1}$ Helen Callaby, ${ }^{2}$ Nicolas Pinto-Sander*, ${ }^{2}$ Suneeta Soni, ${ }^{2}$ Daniel Richardson. ${ }^{1}$ Brighton and Sussex Medical School, Brighton, UK; ${ }^{2}$ Brighton ans Sussex University Hospitals NHS Trust, Brighton, UK

\subsection{6/sextrans-2016-052718.67}

Background Mycoplasma genitalium (MG) caus+es urethritis in males and cervicitis and PID in females. MG prevalence in the UK is not well understood and frequent use of single dose macrolide antibiotics is driving antimicrobial resistance.

Methods From November 2011 to May 2015 selected men with persistent urethritis or proctitis and women with persistent PID 